\title{
Interpretation of the Meaningfulness of Symptom Reduction with Vibegron in Patients with Overactive Bladder: Analyses from EMPOWUR
}

\author{
Jeffrey Frankel - David Staskin · Susann Varano - Michael Kennelly • \\ Diane K. Newman - Matt T. Rosenberg - Rachael A. Jankowich • \\ Denise Shortino · Paul N. Mudd Jr · Cynthia J. Girman
}

Received: August 23, 2021 / Accepted: October 20, 2021 / Published online: December 18, 2021

(C) The Author(s), under exclusive licence to Springer Healthcare Ltd., part of Springer Nature 2021

\begin{abstract}
Introduction: Reductions in bothersome symptoms of overactive bladder (OAB) demonstrate improvement in clinical trials, but patient perception of meaningfulness of such

Supplementary Information The online version contains supplementary material available at https:// doi.org/10.1007/s12325-021-01972-8.
\end{abstract}

J. Frankel

Seattle Urology Research Center, Seattle, WA, USA

D. Staskin

Tufts University School of Medicine, Boston, MA, USA

S. Varano

Clinical Research Consulting, Milford, CT, USA

M. Kennelly

Carolinas Medical Center, Charlotte, NC, USA

D. K. Newman

Perelman School of Medicine, University of

Pennsylvania, Philadelphia, PA, USA

M. T. Rosenberg

Mid-Michigan Health Centers, Jackson, MI, USA

R. A. Jankowich · D. Shortino · P. N. Mudd Jr

Urovant Sciences, Irvine, CA, USA

C. J. Girman $(\bowtie)$

CERobs Consulting, LLC, 2612 Lumina Avenue

North, Wrightsville Beach, Chapel Hill, NC 28480, USA

e-mail: cindy.girman@cerobs.com improvement is lacking. In the 12 -week phase 3 EMPOWUR trial, vibegron significantly reduced average daily number of micturitions, urgency episodes, and urge urinary incontinence (UUI) episodes vs placebo $(P<0.01$ each). This analysis assessed meaningfulness of reductions in clinical endpoints observed in EMPOWUR using patient perception of improvement.

Methods: An anchor-based approach using Patient Global Impression of Change (PGI-C) applied to phase 2 data allowed predefining phase 3 responder definitions. To confirm in phase 3, median change from baseline at week 12 in average daily number of micturitions, urgency episodes, and UUI episodes was generated for each PGI-C category and pooled across treatments. Based on predefined meaningful responder definitions, percentages of patients achieving $\geq 15 \%$ reduction in micturitions (post hoc), $\geq 50 \%$ reduction in urgency episodes (predefined), and $\geq 75 \%$ (predefined) and $\geq 90 \%$ (post hoc) reduction in UUI episodes were determined for patients receiving vibegron or placebo.

Results: Across treatments, for micturitions, urgency episodes, and UUI episodes, median change from baseline to week 12 increased with greater subjective improvement based on PGI-C scores, and median reductions pooled across treatment groups were higher than the responder definitions that patients perceived as improved. Significantly more patients receiving vibegron vs placebo achieved $\geq 15 \%$ reduction 
in micturitions $(56.3 \%$ vs $44.6 \%$, respectively), $\geq 50 \%$ reduction in urgency episodes $(39.5 \%$ vs $32.8 \%), \geq 75 \%$ reduction in UUI episodes $(49.3 \%$ vs $32.8 \%)$, and $\geq 90 \%$ reduction in UUI episodes (35.2\% vs $23.5 \%)$ at week $12(P<0.05$ each $)$.

Conclusion: Significantly more patients treated with vibegron vs placebo in EMPOWUR achieved meaningful reductions in micturitions, urgency episodes, and UUI episodes that were associated with patient-perceived improvement. Results of these analyses support the meaningfulness of reductions in clinical endpoints observed in the 12-week EMPOWUR trial.

Clinical Trials Registration: ClinicalTrials.gov identifier, NCT03492281.

Keywords: Patient-reported outcome; Treatment effectiveness; Urinary incontinence, urge; Patient global impression of change

\section{Key Summary Points}

Why carry out this study?

Standard regulatory endpoints for clinical trials of overactive bladder (OAB) typically include reductions in frequency of micturitions, urgency episodes, and urge urinary incontinence (UUI) episodes to measure improvement; however, these endpoints do not address how meaningful such improvements are to patients

We used an anchor-based approach to interpret the meaningfulness of reduction in micturitions, urgency episodes, and UUI episodes based on Patient Global Impression of Change (PGI-C) from the 12-week, phase 3, randomized, controlled EMPOWUR trial of vibegron vs placebo in patients with $\mathrm{OAB}$

\section{What was learned from study?}

Across treatment groups, patients who experienced improvement based on PGI-C had greater reductions from baseline at week 12 in $\mathrm{OAB}$ outcomes
The magnitude of improvement in $\mathrm{OAB}$ outcomes associated with patientperceived improvement was $\geq 15 \%$ reduction in micturitions, $\geq 50 \%$ reduction in urgency episodes, and $\geq 75 \%$ and $\geq 90 \%$ reduction in UUI episodes

A significantly greater percentage of patients receiving vibegron vs placebo achieved meaningful reductions in $\mathrm{OAB}$ outcomes based on thresholds defined using patient impression of improvement

\section{INTRODUCTION}

Overactive bladder $(\mathrm{OAB})$ is characterized by episodes of a strong, sudden urge or need to urinate immediately (urgency), usually accompanied by frequency and nocturia with or without urge urinary incontinence (UUI) $[1,2]$. This common and chronic condition can have a profound impact on patient's lives by causing embarrassment, impairing daily functioning and relationships, and in some cases requiring patients to plan their lives around accommodating their symptoms [3-5]. Accordingly, OAB has been shown to negatively affect healthrelated quality of life (QoL) and contribute to symptoms of anxiety and depression [6-8]. Among patients with $\mathrm{OAB}$, the severity of $\mathrm{OAB}$ symptoms is positively correlated with the severity of anxiety and depression symptoms $[9,10]$.

Goals of treatment for $\mathrm{OAB}$ include improving not only symptom control but also patient QoL [2]. Standard regulatory endpoints, including reductions in bothersome symptoms (frequent micturitions, urgency episodes, and UUI episodes), are typically collected and reported in clinical trials of OAB [11-15]. Although clinical trials are increasingly including patient-reported outcomes (PROs) to measure symptoms and impact on daily functioning [11-14, 16, 17], it is important to individualize goal setting with the understanding that improvements in symptoms and QoL observed in large clinical trials may not necessarily translate to meaningful changes for individual 
patients. Assessing the magnitude of improvement in bothersome symptoms that is considered relevant from the patient perspective represents an important step toward establishing the ability of interventions to produce meaningful changes. For example, even small improvements in $\mathrm{OAB}$ symptoms, such as reduction in micturition frequency by one or two episodes, may be considered meaningful by an individual patient. Several trials of $O A B$ medications have incorporated responder rates in an effort to demonstrate rates of clinically meaningful change based on predefined endpoints [12, 14, 17-19].

Vibegron is a selective $\beta_{3}$-adrenergic receptor agonist approved for treatment of $\mathrm{OAB}$ in adults [20]. In the 12-week, phase 3 EMPOWUR trial, vibegron significantly reduced micturitions and UUI episodes (coprimary endpoints), as well as urgency episodes (key secondary endpoint), vs placebo $(P<0.01$ each $)$ [21]. Compared with placebo, vibegron was also associated with significantly greater improvement in PROs, including the $\mathrm{OAB}$ questionnaire $(\mathrm{OAB}-\mathrm{q})$ and Patient Global Impression scores [17]. Statistically significantly greater proportions of patients receiving vibegron vs placebo were classified as responders at week 12 for coping scores and symptom bother scores based on a 10-point minimally important difference (MID) for OAB-q subscales.

The current analysis used an anchor-based approach to interpret the meaningfulness of reductions in clinical trial endpoints observed in the EMPOWUR trial and to demonstrate treatment effects in the proportion of patients achieving meaningful change in $\mathrm{OAB}$ endpoints, with the threshold predefined based on categories of improvement on the Patient Global Impression of Change (PGI-C).

\section{METHODS}

\section{Study Design and Patients}

Detailed methods of the EMPOWUR trial have previously been published [21]. Briefly, the trial consisted of a 2-week single-blind placebo runin period, a 12-week double-blind treatment period, and a 4-week safety follow-up period. Adults with $\mathrm{OAB}$ wet (defined as an average of $\geq 8.0$ micturitions and $\geq 1.0$ UUI episodes per day in the 7-day voiding diary at baseline) or $O A B$ dry (defined as an average of $\geq 8$ micturitions, $\geq 3.0$ urgency episodes, and $<1.0$ UUI episode per day in the 7-day voiding diary at baseline) for $\geq 3$ months before the screening visit were randomly assigned in a 5:5:4 ratio to receive once-daily vibegron $75 \mathrm{mg}$, placebo, or tolterodine $4 \mathrm{mg}$ extended release for 12 weeks. Patients completed 7-day diaries, which have been previously validated [22], at baseline and at weeks $2,4,8$, and 12 , documenting micturitions, urgency, and incontinence and whether incontinence episodes were due to urgency or other reasons. Among other assessments, patients completed the PGI-C at baseline and at weeks 4,8 , and 12 [17]. The commonly used PGI-C assesses patient perception of change since the start of the study, scored from 1 to 7 , with lower scores indicating greater improvement [23]. The EMPOWUR trial [21] was conducted in accordance with International Conference on Harmonisation guidelines for Good Clinical Practice. The study protocol, amendments, and written materials provided to patients, including the informed consent form, were approved at each study site by the local institutional review boards, independent ethics committees, or research ethics boards and by the central institutional review board (IRB), Copernicus Group IRB (Cary, North Carolina); all patients provided written informed consent.

\section{Determination of Clinically Meaningful Cutoff Values}

Determination of clinically meaningful cutoff values was based on anchor-based analyses of phase 2 trial data [24] and further confirmed using phase 3 trial data. For the anchor-based analyses, median change of each clinical trial endpoint was derived for each category of change on the PGI-C, which served as the anchor. As supportive evidence, qualitative patient interviews were performed in August 2017 among adult patients with OAB to identify responder-based endpoints reflecting clinically 
meaningful improvement. The study protocol, discussion guide, and informed consent forms for the patient interviews were approved by WCG IRB (Puyallup, WA). Participants were recruited by trained medical recruiters and were eligible for the qualitative interviews if they had been diagnosed with $\mathrm{OAB}$ wet or with $\mathrm{OAB}$ dry for $\geq 3$ months before screening and were able to read and speak English. Patients were asked to describe their $\mathrm{OAB}$ symptoms and identify the most bothersome symptom, with subsequent questions focusing on determining meaningful changes in $\mathrm{OAB}$ symptoms from the patient perspective. Dominant themes were compared across interviews to identify patterns in descriptions of OAB symptoms and perceptions related to meaningful improvement. Patients were then asked to estimate the number of times they typically experienced each bothersome symptom in a 24 -h period and to describe the amount of improvement in each symptom (either as a reduction in number of episodes or as a relative reduction) that they would consider meaningful enough to continue taking a medication.

Using anchor-based methodology in phase 2 and qualitative results of patient interviews, the following cutoff values were selected and predefined in EMPOWUR as representing clinically meaningful improvement: $\geq 50 \%$ reduction in urgency episodes and $\geq 75 \%$ reduction in UUI episodes from baseline at week 12 [21]. These thresholds were derived by assessing the magnitude of change in each $\mathrm{OAB}$ endpoint that was associated with moderately to much better responses on the PGI-C.

\section{Statistical Analysis}

The median change from baseline at week 12 in average daily number of micturitions, urgency episodes, and UUI episodes was generated for four PGI-C categories (no change or worse [consisting of much worse, moderately worse, a little worse, no change], a little better, moderately better, and much better) pooled across treatments. The full analysis set (FAS), defined as all randomized patients with $\geq 1$ evaluable change from baseline measurement for micturitions, was used for analysis of micturitions and urgency episodes; the FAS for incontinence (FAS-I), which included all randomized patients with $\mathrm{OAB}$ wet at baseline who had $\geq 1$ evaluable change from baseline measurement for UUI episodes, was used for analysis of UUI episodes.

Analyses of predefined key secondary endpoints in EMPOWUR based on responder definitions described above were performed by deriving the percentages of patients achieving $\geq 50 \%$ reduction in urgency episodes and $\geq 75 \%$ reduction in UUI episodes for each treatment group. In addition, the percentages of patients achieving $\geq 15 \%$ reduction in micturitions (post hoc) and $\geq 90 \%$ (post hoc) reduction in UUI episodes were determined. The difference in proportion between treatment groups and corresponding 95\% CIs and $P$ values were calculated using Cochran-Mantel-Haenszel $(\mathrm{CMH})$ risk difference estimates stratified by OAB type (wet vs dry; for urgency and micturitions) and sex, with weights proposed by Greenland and Robins [25]. For the post hoc analyses $(\geq 15 \%$ reduction in micturitions and $\geq 90 \%$ reduction in UUI episodes), no adjustments for multiplicity were performed. Nominal $P$ values are provided for descriptive purposes. For the predefined key secondary endpoints, the type 1 error rate was controlled at the 0.05 level using a stepwise gate-keeping procedure over the coprimary and key secondary hypotheses. Multiple imputations methods were used to estimate missing values for the responder endpoints and to estimate the percentage of responders for each treatment.

\section{RESULTS}

\section{Patients}

As previously reported, of 1518 patients enrolled in EMPOWUR and randomly assigned to vibegron $(N=547)$, placebo $(N=540)$, or tolterodine $(N=431), 1463$ were included in the FAS (vibegron, $N=526$; placebo, $N=520$; tolterodine, $N=417)$, and 1127 were included in the FAS-I (vibegron, $N=403$; placebo, $N=405$; tolterodine, $N=319$ ) [21]. In the overall FAS 
Table 1 Demographics and baseline clinical characteristics (FAS)

\begin{tabular}{|c|c|c|c|c|}
\hline Characteristic & $\begin{array}{l}\text { Placebo } \\
(N=520)\end{array}$ & $\begin{array}{l}\text { Vibegron } \\
(N=526)\end{array}$ & $\begin{array}{l}\text { Tolterodine } \\
(N=417)\end{array}$ & $\begin{array}{l}\text { Overall } \\
(N=1463)\end{array}$ \\
\hline Mean (SD) age, years & $59.9(13.3)$ & $60.8(13.3)$ & $59.8(13.2)$ & $60.2(13.28)$ \\
\hline Women, $n(\%)$ & $445(85.6)$ & $449(85.4)$ & $352(84.4)$ & $1246(85.2)$ \\
\hline \multicolumn{5}{|l|}{ OAB type, $n(\%)$} \\
\hline Wet & $405(77.9)$ & $403(76.6)$ & $319(76.5)$ & $1127(77.0)$ \\
\hline Dry & $115(22.1)$ & $123(23.4)$ & $98(23.5)$ & $336(23.0)$ \\
\hline Median (IQR) micturitions per day ${ }^{a}$ & $10.4(9.2-13.1)$ & $10.4(9.0-12.6)$ & $10.7(9.1-12.9)$ & $10.6(9.1-12.9)$ \\
\hline $\begin{array}{l}\text { Median (IQR) urgency episodes per } \\
\text { day }^{a}\end{array}$ & $8.0(4.6-10.5)$ & $7.8(4.6-10.7)$ & $8.0(4.9-10.3)$ & $7.9(4.7-10.6)$ \\
\hline $\begin{array}{l}\text { Median (IQR) UUI episodes per } \\
\text { day }^{\text {a,b }}\end{array}$ & $2.5(1.6-4.4)$ & $2.6(1.6-4.1)$ & $2.4(1.7-4.6)$ & $2.6(1.6-4.4)$ \\
\hline Median (range) PGI-C ${ }^{c}$ & $4.0(1.0-7.0)$ & $4.0(1.0-7.0)$ & $4.0(1.0-7.0)$ & $4.0(1.0-7.0)$ \\
\hline
\end{tabular}

All randomized patients who took $\geq 1$ dose of double-blind study treatment and had $\geq 1$ evaluable change from baseline micturition measurement

FAS full analysis set, FAS-I FAS for incontinence, IQR interquartile range, $O A B$ overactive bladder, $P G I-C$ Patient Global Impression of Change, UUI urge urinary incontinence

${ }^{a}$ Defined as the average daily number of episodes derived from the 7-day voiding diary

${ }^{\mathrm{b}}$ Analyzed in the FAS-I, which included all randomized patients who took $\geq 1$ dose of double-blind study treatment and had $\geq 1$ evaluable change from baseline measurement for UUI episodes. Placebo, $N=405$; vibegron, $N=403$; tolterodine, $N=319$

'Placebo, $N=519$; vibegron, $N=525$; tolterodine, $N=417$

population, $85 \%$ of patients were women, $78 \%$ were white, and $43 \%$ were $\geq 65$ years old (Table 1). At baseline, patients in the FAS had a median (interquartile range [IQR]) of 10.6 (9.1-12.9) micturitions and 7.9 (4.7-10.6) urgency episodes per day on average, and those in the FAS-I had a median (IQR) of 2.6 (1.6-4.4) UUI episodes per day on average.

\section{Outcomes}

For each endpoint (micturitions, urgency episodes, and UUI episodes), the median change from baseline to week 12 increased with greater subjective improvement on PGI-C scores. Across treatment groups, patients reporting moderately better for PGI-C had a median percentage reduction from baseline in micturitions of $18.2 \% \quad$ (i.e., $\geq 15 \%$; Fig. $1 \mathrm{~A}$; Supplementary
Table 1). A statistically significantly greater percentage of patients receiving vibegron $(56.3 \%)$ vs placebo $(44.6 \%)$ achieved $\geq 15 \%$ reduction from baseline (corresponding to PGI$\mathrm{C}$ of moderately better) in micturitions $(\mathrm{CMH}$ difference [95\% CI], 11.8 [5.7-17.9]; nominal $P<0.001$; Fig. 1B). A statistically significantly greater percentage of patients receiving tolterodine $(53.2 \%)$ vs placebo achieved $\geq 15 \%$ reduction from baseline in micturitions $(\mathrm{CMH}$ difference [95\% CI], 8.8 [2.3-15.3]; nominal $P<0.01$; Fig. 1B).

Overall, patients reporting moderately or much better for PGI-C had a median percentage reduction from baseline in urgency episodes of $35.0 \%$ or $60.9 \%$ (i.e., $\geq 50 \%$; Fig. 2 A; Supplementary Table 1 ), respectively. Statistically significantly more patients receiving vibegron $(39.5 \%)$ vs placebo (32.8\%) achieved $\geq 50 \%$ reduction from baseline (corresponding to PGI-C 
of much better) in urgency episodes (CMH difference $[95 \%$ CI], $6.8 \quad[0.9-12.7] ; \quad P<0.05$; Fig. 2B). No significant differences were seen in the percentage of patients achieving $\geq 50 \%$ reduction from baseline in urgency episodes between tolterodine (36.4\%) and placebo $(\mathrm{CMH}$ difference [95\% CI], 3.7 [ -2.5 to 10.0$] ; P>0.05$; Fig. 2B).

Patients reporting moderately or much better for PGI-C had a median percentage reduction from baseline in UUI episodes of $64.5 \%$ or $93.2 \%$ (i.e., $\geq 75 \%$ and $\geq 90 \%$; Fig. 3A; Supplementary Table 1), respectively. Similar to the statistically significantly greater percentage of patients experiencing $a \geq 75 \%$ reduction in UUI episodes with vibegron vs placebo reported
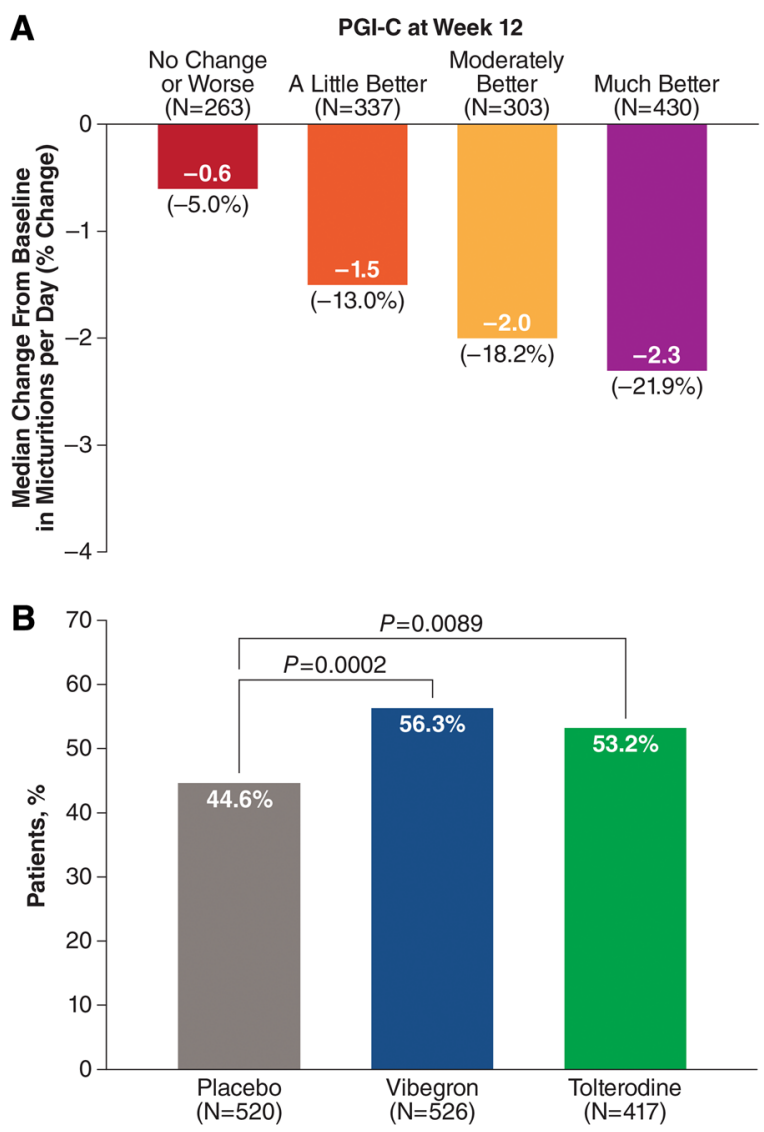

Fig. 1 A Median (median percentage) change from baseline at week 12 in mean daily number of micturitions by PGI-C and B percentage of patients achieving $\geq 15 \%$ reduction from baseline in mean daily number of micturitions. PGI-C Patient Global Impression of Change in EMPOWUR $(49.3 \%$ vs $32.8 \%$, respectively; $\mathrm{CMH}$ difference $\left[\begin{array}{lll}95 \% & \mathrm{CI}\end{array}\right], 16.5$ [9.7-23.4]; $P<0.0001)$, statistically significantly more patients receiving vibegron vs placebo experienced $\mathrm{a} \geq 90 \%$ reduction from baseline (corresponding to PGI-C of much better) in UUI episodes $(35.2 \%$ vs $23.5 \%$, respectively; $\mathrm{CMH}$ difference [95\% CI], 11.7 [5.2-18.1]; nominal $P<0.001$; Fig. 3B). Statistically significantly more patients receiving tolterodine $(30.6 \%)$ vs placebo experienced $a \geq 90 \%$ reduction from baseline in UUI episodes (CMH difference [95\% CI], 7.1 [0.3-13.8]; nominal $P<0.05$; Fig. 3B).
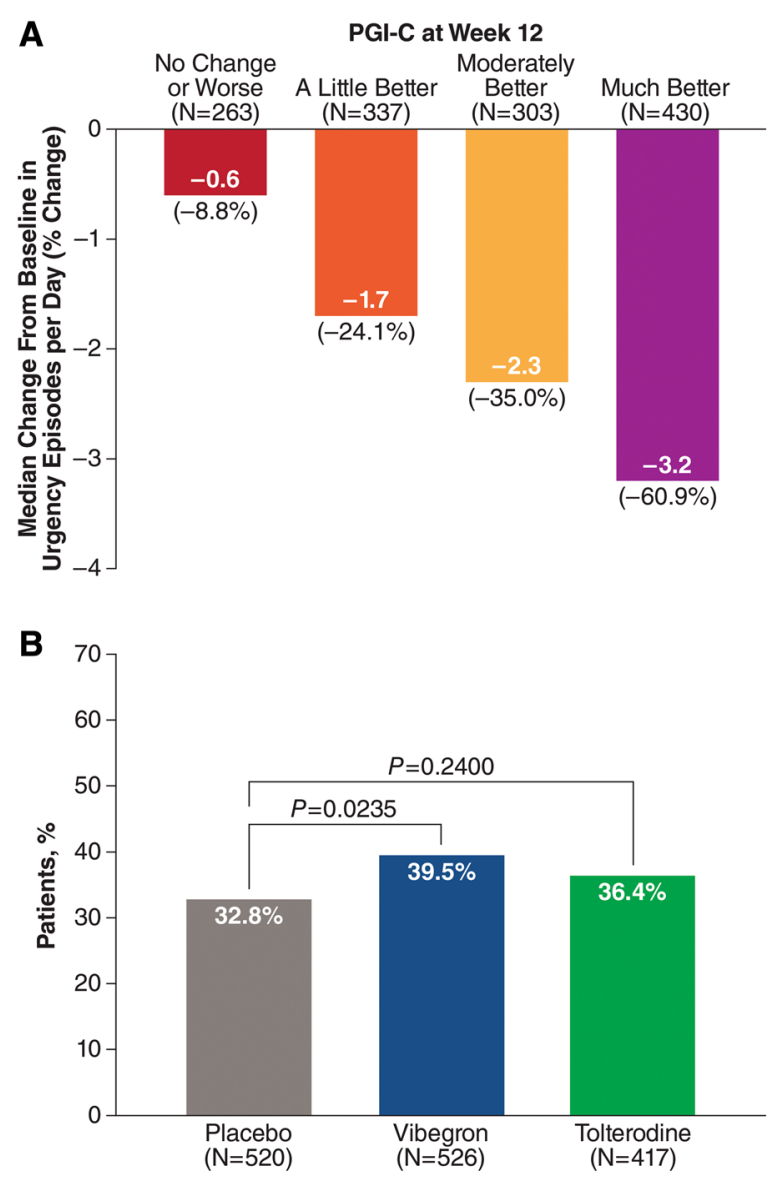

Fig. 2 A Median (median percentage) change from baseline at week 12 in mean daily number of urgency episodes by PGI-C and B percentage of patients achieving $\geq 50 \%$ reduction from baseline in mean daily number of urgency episodes. PGI-C Patient Global Impression of Change 


\section{Supportive Patient Interviews}

Qualitative patient interviews were conducted among 11 patients ( 8 with $\mathrm{OAB}$ wet and 3 with OAB dry; 8 female/ 3 male; mean age, 50.2 years; 8 white). Symptoms of OAB considered most bothersome included urinary frequency, urgency, and leakage. All 11 patients indicated that a 25 to $50 \%$ reduction in total number of micturitions would represent important improvement; 9 of 11 reported that a $50 \%$ reduction in urgency episodes would be an important improvement, with an additional patient saying that a reduction of this magnitude "may" be meaningful. Of the eight patients with $\mathrm{OAB}$ wet, six indicated that they would find a $70 \%$ reduction in UUI episodes to be a meaningful improvement. Additionally, patients agreed that each one-category shift in PGI questions would represent meaningful improvement; this degree of change is further supported by correlations between Patient Global Impression of Improvement (corresponding to PGI-C) scores and health-related QoL outcomes [23].

\section{DISCUSSION}

In the phase 3 EMPOWUR trial, vibegron $75 \mathrm{mg}$ demonstrated consistent mean improvements across clinically relevant $\mathrm{OAB}$ symptoms, including micturition frequency, urgency episodes, and UUI episodes compared with placebo [21]. The clinical relevance of these improvements is supported by significant changes in PROs with vibegron vs placebo, including improvements in the OAB-q subscales and PGIC [17].

In response to a regulatory request, the current analyses were performed to provide validation for the clinical meaningfulness of coprimary and key secondary efficacy endpoints from the EMPOWUR trial. Associations between median percentage changes in frequency of average daily micturitions, urgency episodes, and UUI episodes and PGI-C categories were moderate to strong, further supporting use of the PGI-C in deriving responder definitions [26]. Notably, the cutoff values used were
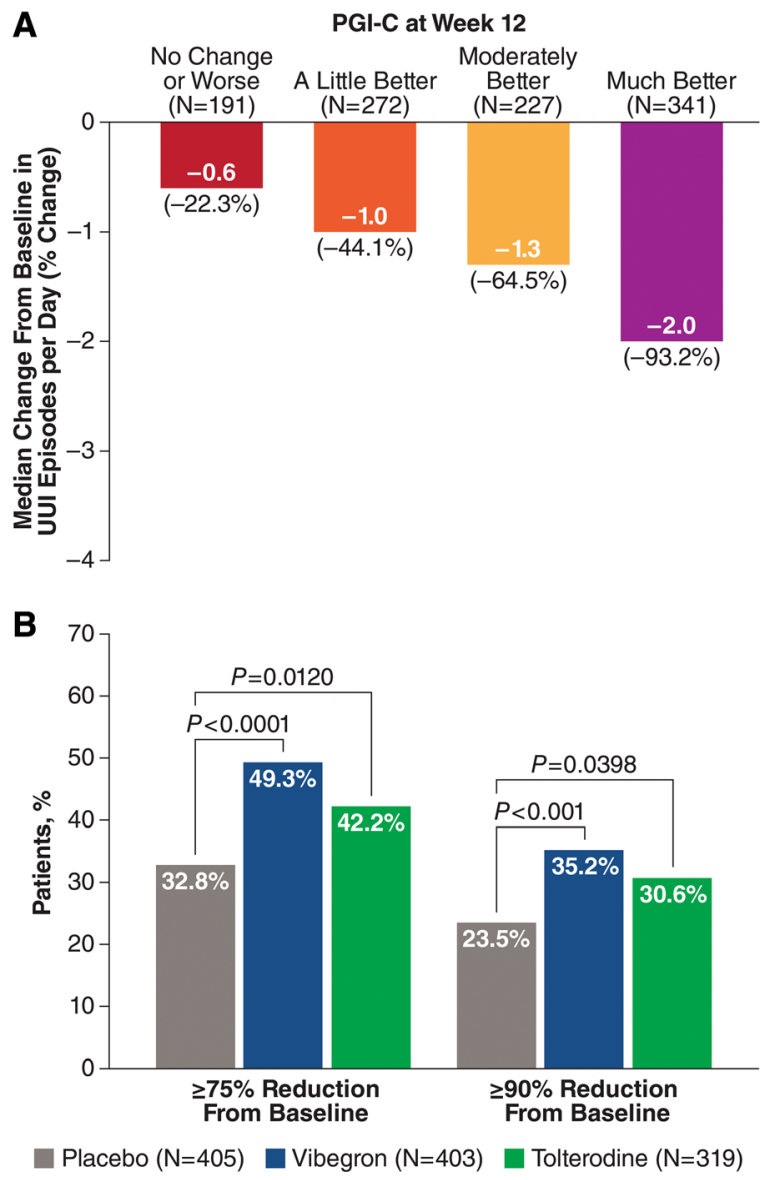

Fig. 3 A Median (median percentage) change from baseline at week 12 in mean daily number of UUI episodes by PGI-C and $\mathbf{B}$ percentage of patients achieving $a \geq 75 \%$ reduction and $a \geq 90 \%$ reduction from baseline in mean daily number of UUI episodes. PGI-C Patient Global Impression of Change, UUI urge urinary incontinence

comparable to or greater than those identified as representing minimally important differences in an analysis that employed anchor- and distribution-based methods using data from the phase 2, 8-week, randomized, placebo- and active-controlled trial [27]. These responder definitions derived from the phase 2 trial allowed prespecifying phase 3 responder definitions in EMPOWUR. In the current analysis, significantly more patients receiving vibegron vs placebo in EMPOWUR achieved meaningful reductions in micturitions, urgency episodes, and UUI episodes that were associated with 
patient-perceived improvement according to PGI-C. Results of this analysis are strongly supportive of the clinical meaningfulness of the coprimary and key secondary endpoint results from the EMPOWUR trial. In addition, qualitative interview results identified micturition frequency, urgency, and leakage as the most bothersome symptoms, providing further support for the content validity of the patient voiding diary and efficacy endpoints used in the phase 3 EMPOWUR trial of vibegron in patients with $\mathrm{OAB}$ [21]. In addition, such interviews supported the responder definitions for UUI and urgency episodes that were prespecified in EMPOWUR as key secondary endpoints.

Clinical trials of OAB have evaluated clinical meaningfulness of interventions using a variety of approaches. Demonstration of statistically significant effects on PROs-including measures of QoL, patient satisfaction with treatment, and patient perception of improvement-reflect treatment benefits in relation to outcomes of importance from the patient perspective $[11,13,14,17]$. Anchor- and distribution-based methods can be used to determine thresholds for responder analyses to demonstrate clinically meaningful improvement in PROs or objective measures [17-19, 28, 29]. Previous studies have reported responder rates based on percentage changes in urgency episodes and/or incontinence episodes [12, 14, 21]. Notably, a pooled analysis of three randomized, placebocontrolled trials of darifenacin in adults with $\mathrm{OAB}$ reported responder rates for $\mathrm{a} \geq 50 \%$ reduction in urgency episodes (31-34\%) and a $\geq 90 \%$ reduction in incontinence episodes (27-28\%) with darifenacin [12]; corresponding placebo rates were lower in the darifenacin analyses $(23-24 \%$ and $17 \%$, respectively), resulting in similar treatment differences vs placebo. The current analysis adds to the literature by identifying cutoff values that are associated with patient perception of meaningful improvement and by demonstrating a significant drug effect based on these cutoff values.

Correlations between $\mathrm{OAB}$ symptoms and PROs support the use of PROs as a measure of treatment efficacy and provide an indication of the degree to which improvements in $\mathrm{OAB}$ symptoms may predict improvements in patient perceptions and health-related QoL. Analyses of data from several clinical trials of anticholinergics demonstrated that improvements in OAB symptoms over 12 weeks of treatment were significantly correlated with improvements in Patient Perception of Bladder Condition (PPBC) as well as with improvements in health-related QoL [18, 30-33]. Correlation sizes were typically moderate for PPBC and small to moderate for health-related QoL domains.

Patient satisfaction is often driven by patient expectations and by whether the benefits of treatment meet patient goals [34]. Goal setting is an individualized process that addresses the ways in which a health condition affects the patient's daily life. Patient goals may refer to improvement in symptoms (e.g., reducing nocturia or urgency), although specific goals may also be stated in relation to activities (e.g., improving sleep quality, being able to walk the dog without needing the toilet) [4, 34, 35]. Studies have demonstrated statistically significant correlations between goal achievement and patient satisfaction as well as between goal achievement and both patient perception of treatment benefit and health-related QoL among patients with $\mathrm{OAB}$ receiving anticholinergic therapy $[35,36]$. Although it is reasonable to expect that the significantly higher response rates achieved with vibegron vs placebo in the current analysis would translate to greater levels of goal attainment and patient satisfaction, further research is warranted.

Placebo-controlled trials are critical to interpretation of $\mathrm{OAB}$ treatment effects because of the typically strong placebo and learning effects in clinical trials. In a meta-analysis of 57 randomized, placebo-controlled trials investigating oral pharmacotherapy for $\mathrm{OAB}$, placebo was associated with statistically significant improvements from baseline in mean [95\% CI] daily numbers of micturitions $(-0.45[-0.51$ to $-0.40] ; \quad P<0.001)$, urgency episodes $(-0.50$ $[-0.62$ to -0.40$] ; P<0.001)$, and UUI episodes $(-0.46[-0.55$ to -0.38$] ; P<0.001)$ [37]. The authors noted several potential factors underlying the placebo response including the use of voiding diaries, which may be a form of behavioral therapy; contextual factors; a 
psychological response to being in a perceived therapeutic situation; effects of information provided to patients during studies; and the dynamic nature of OAB [37]. Assessing eligibility after patients have completed the voiding diary at screening and during the placebo runin period, as was done in the EMPOWUR trial, is important to reduce the potential placebo effect associated with maintaining a voiding diary. However, additional factors may contribute to the placebo effect, and it is notable that approximately 24 to $45 \%$ of patients receiving placebo in this analysis were classified as responders based on the various cutoff criteria.

Key limitations of the current analysis are that it did not separately analyze patients with $\mathrm{OAB}$ wet vs $\mathrm{OAB}$ dry. These analyses were not powered to detect statistical significance; sample size calculations were based on the coprimary endpoints. Additionally, the findings represent results from clinical trials, which may not be generalizable to real-world settings where the population is more heterogenous and where lifestyle factors may affect patient perceptions of improvement.

\section{CONCLUSIONS}

Although $\mathrm{OAB}$ is highly prevalent among adults, large clinical studies often neglect to report patient perception of meaningfulness of symptomatic improvement. Results of these analyses support the clinical meaningfulness of the reductions in clinical endpoints observed in the 12-week EMPOWUR trial; significantly more patients treated with vibegron vs placebo achieved meaningful reductions in micturitions, urgency episodes, and UUI episodes that were associated with patient-perceived improvement, suggesting that such statistically significant improvements are indeed meaningful to patients. Clinically meaningful improvements in bothersome symptoms of OAB would be expected to help both the provider and patient achieve treatment goals and contribute to greater patient satisfaction and treatment persistence. Vibegron therefore represents an important new treatment option with meaningful clinical benefit for patients with $\mathrm{OAB}$.

\section{ACKNOWLEDGEMENTS}

We thank the participants of the study.

Funding. Funding for this study, including funding for the journal's rapid service and open access fees and for medical writing and editorial support, was provided by Urovant Sciences (Irvine, CA, USA).

Authorship. All named authors meet the International Committee of Medical Journal Editors (ICMJE) criteria for authorship for this article, take responsibility for the integrity of the work as a whole, and have given their approval for this version to be published.

Author contributions. Rachael A. Jankowich, Denise Shortino, Paul N. Mudd Jr, and Cynthia J. Girman contributed to the conception and design of the present analyses. Jeffrey Frankel, David Staskin, and Susann Varano were investigators for the EMPOWUR trial, from which the data were derived. All named authors participated in analysis and interpretation of the data. The first draft of the manuscript was written by a medical writer under the direction of all authors, and all authors commented on previous versions of the manuscript and have read and approved the final version.

Medical writing, editorial, and other assistance. Medical writing and editorial support was provided by Adrienne Drinkwater, $\mathrm{PhD}$, for The Curry Rockefeller Group, LLC (Tarrytown, NY, USA), and was funded by Urovant Sciences (Irvine, CA, USA).

Disclosures. Jeffrey Frankel is an advisor for Urovant Sciences; a meeting participant/lecturer for Astellas, Myovant, Pfizer, and Urovant Sciences; and is an investigator for Astellas, Exact Sciences, Johnson \& Johnson, and Pfizer. David Staskin is an advisor for Astellas, UroCure, and Urovant Sciences; a meeting participant/lecturer and investigator for Astellas and Urovant Sciences; and holds other interests in UroCure. Susann Varano is a consultant and speaker for Urovant Sciences, a principal investigator for Clinical Research Consulting and 
holds academic positions at Sacred Heart University and University of Bridgeport. Michael Kennelly is an advisor for AbbVie, Astellas, Boston Scientific, Coloplast, Laborie, and Urovant Sciences and has received study funding from Abbvie, Amphora, Astellas, Axonics, Boston Scientific, Coloplast, Cook Myosite, Dignify Therapeutics, EBT Medical, FemPulse, Ipsen, Taris, and Uro1. Diane K. Newman is an advisor for Digital Science Press, EBT Medical, and Urovant Sciences; and has received funding from the National Institutes of Health. Matt T. Rosenberg is an advisor to and meeting participant/lecturer for Astellas and Urovant Sciences, is an advisor to Sanofi, and is a meeting participant/lecturer for Ferring. Rachael A. Jankowich, Denise Shortino, and Paul N. Mudd Jr were employees of Urovant Sciences at the time the work was conducted and are now employees of Priovant Therapeutics, Inc. Cynthia J. Girman is the founder and President of CERobs Consulting, which was contracted by Urovant to provide consulting.

Compliance with ethics guidelines. The EMPOWUR trial was conducted in accordance with International Conference on Harmonisation guidelines for Good Clinical Practice. The study protocol, amendments, and written materials provided to patients, including the informed consent form, were approved at each study site by the local institutional review boards, independent ethics committees, or research ethics boards and by the central institutional review board (IRB), Copernicus Group IRB (Cary, NC); all patients provided written informed consent. The study protocol, discussion guide, and informed consent forms for the patient interviews were approved by WCG IRB (Puyallup, WA).

Data availability. The datasets generated during and/or analyzed during the current study are available from the from the study sponsor, Urovant Sciences (email: medinfo@urovant.com), on reasonable request.

Prior presentation. These data have been previously presented at the South Central Section of the American Urological Society
(Scottsdale, AZ, USA; September 29-October 2, 2021) as well as additional encore presentations.

Open Access. This article is licensed under a Creative Commons Attribution-NonCommercial 4.0 International License, which permits any non-commercial use, sharing, adaptation, distribution and reproduction in any medium or format, as long as you give appropriate credit to the original author(s) and the source, provide a link to the Creative Commons licence, and indicate if changes were made. The images or other third party material in this article are included in the article's Creative Commons licence, unless indicated otherwise in a credit line to the material. If material is not included in the article's Creative Commons licence and your intended use is not permitted by statutory regulation or exceeds the permitted use, you will need to obtain permission directly from the copyright holder. To view a copy of this licence, visit http://creativecommons.org/licenses/by$\mathrm{nc} / 4.0 /$.

\section{REFERENCES}

1. Haylen BT, de Ridder D, Freeman RM, et al. An International Urogynecological Association (IUGA)/International Continence Society (ICS) joint report on the terminology for female pelvic floor dysfunction. Neurourol Urodyn. 2010;29(1): $4-20$.

2. Gormley EA, Lightner DJ, Burgio KL, et al. Diagnosis and treatment of overactive bladder (nonneurogenic) in adults: AUA/SUFU guideline. American Urological Association; 2019.

3. Coyne KS, Sexton CC, Vats V, Thompson C, Kopp ZS, Milsom I. National community prevalence of overactive bladder in the United States stratified by sex and age. Urology. 2011;77(5):1081-7.

4. Rantell A, Cardozo L, Khullar V. Personal goals and expectations of OAB patients in the UK. Neurourol Urodyn. 2017;36(4):1194-200.

5. Kinsey D, Pretorius S, Glover L, Alexander T. The psychological impact of overactive bladder: a systematic review. J Health Psychol. 2016;21(1):69-81.

6. Coyne KS, Payne C, Bhattacharyya SK, et al. The impact of urinary urgency and frequency on health- 
related quality of life in overactive bladder: results from a national community survey. Value Health. 2004;7(4):455-63.

7. Coyne KS, Sexton CC, Kopp ZS, Ebel-Bitoun C, Milsom I, Chapple C. The impact of overactive bladder on mental health, work productivity and health-related quality of life in the UK and Sweden: results from EpiLUTS. BJU Int. 2011;108(9): 1459-71.

8. Johnston KM, Walker DR, Lakzadeh P. Characterizing the health-related quality of life burden of overactive bladder using disease-specific patientreported outcome measures: a systematic literature review. Adv Ther. 2019;36(3):548-62.

9. Lai HH, Rawal A, Shen B, Vetter J. The relationship between anxiety and overactive bladder or urinary incontinence symptoms in the clinical population. Urology. 2016;98:50-7.

10. Lai HH, Shen B, Rawal A, Vetter J. The relationship between depression and overactive bladder/urinary incontinence symptoms in the clinical $\mathrm{OAB}$ population. BMC Urol. 2016;16(1):60.

11. Appell RA. Clinical efficacy and safety of tolterodine in the treatment of overactive bladder: a pooled analysis. Urology. 1997;50(6A Suppl):90-9.

12. Chapple C, Steers W, Norton P, et al. A pooled analysis of three phase III studies to investigate the efficacy, tolerability and safety of darifenacin, a muscarinic M3 selective receptor antagonist, in the treatment of overactive bladder. BJU Int. 2005;95(7):993-1001.

13. Nitti VW, Dmochowski R, Herschorn S, et al. OnabotulinumtoxinA for the treatment of patients with overactive bladder and urinary incontinence: results of a phase 3 , randomized, placebo controlled trial. J Urol. 2013;189(6):2186-93.

14. Nitti VW, Khullar V, van Kerrebroeck P, et al. Mirabegron for the treatment of overactive bladder: a prespecified pooled efficacy analysis and pooled safety analysis of three randomised, double-blind, placebo-controlled, phase III studies. Int J Clin Pract. 2013;67(7):619-32.

15. Chapple CR, Kelleher CJ, Evans CJ, et al. A narrative review of patient-reported outcomes in overactive bladder: what is the way of the future? Eur Urol. 2016;70(5):799-805.

16. Brubaker L, Chapple C, Coyne KS, Kopp Z. Patientreported outcomes in overactive bladder: importance for determining clinical effectiveness of treatment. Urology. 2006;68(2 Suppl):3-8.
17. Frankel J, Varano S, Staskin D, Shortino D, Jankowich R, Mudd PN Jr. Vibegron improves quality-oflife measures in patients with overactive bladder: patient-reported outcomes from the EMPOWUR study. Int J Clin Pract. 2021;75(5):e13937.

18. Abrams P, Kelleher C, Huels J, Quebe-Fehling E, Omar MA, Steel M. Clinical relevance of health-related quality of life outcomes with darifenacin. BJU Int. 2008;102(2):208-13.

19. Herschorn S, Staskin D, Tu LM, et al. Patient-reported outcomes in patients with overactive bladder treated with mirabegron and tolterodine in a prospective, double-blind, randomized, two-period crossover, multicenter study (PREFER). Health Qual Life Outcomes. 2018;16(1):69.

20. GEMTESA $^{\circledR}$ (vibegron). Full Prescribing Information. Irvine: Urovant Sciences; 2020.

21. Staskin D, Frankel J, Varano S, Shortino D, Jankowich R, Mudd PN Jr. International phase III, randomized, double-blind, placebo and active controlled study to evaluate the safety and efficacy of vibegron in patients with symptoms of overactive bladder: EMPOWUR. J Urol. 2020;204(2): 316-24.

22. Brown JS, McNaughton KS, Wyman JF, et al. Measurement characteristics of a voiding diary for use by men and women with overactive bladder. Urology. 2003;61(4):802-9.

23. Tincello DG, Owen RK, Slack MC, Abrams KR. Validation of the Patient Global Impression scales for use in detrusor overactivity: secondary analysis of the RELAX study. BJOG. 2013;120(2):212-6.

24. Mitcheson HD, Samanta S, Muldowney K, et al. Vibegron (RVT-901/MK-4618/KRP-114V) administered once daily as monotherapy or concomitantly with tolterodine in patients with an overactive bladder: a multicenter, phase IIb, randomized, double-blind, controlled trial. Eur Urol. 2019;75(2): 274-82.

25. Greenland S, Robins JM. Estimation of a common effect parameter from sparse follow-up data. Biometrics. 1985;41(1):55-68.

26. Norquist JM, Girman C, Fehnel S, DeMuro-Mercon C, Santanello N. Choice of recall period for patientreported outcome (PRO) measures: criteria for consideration. Qual Life Res. 2012;21(6):1013-20.

27. Pinto CA, Norquist J, Liao J, Frenkl T, Girman C. Understanding MID for micturition frequency, a pivotal endpoint for $\mathrm{OAB}$ studies. Presented at: ISPOR 19th Annual International Meeting; May 31-June 4, 2014; Montreal, QC, Canada. 
28. US Department of Health and Human Services. Guidance for Industry: Patient-Reported Outcome Measures: Use in Medical Product Development to Support Labeling Claims. Silver Spring, MD: US Department of Health and Human Services; 2009.

29. Desroziers K, Aballéa S, Maman K, Nazir J, Odeyemi I, Hakimi Z. Estimating EQ-5D and OAB-5D health state utilities for patients with overactive bladder. Health Qual Life Outcomes. 2013;11:200.

30. Coyne KS, Elinoff V, Gordon DA, et al. Relationships between improvements in symptoms and patient assessments of bladder condition, symptom bother and health-related quality of life in patients with overactive bladder treated with tolterodine. Int J Clin Pract. 2008;62(6):925-31.

31. Crosby RD, Mathias SD, Marshall TS. Relationships between symptoms, symptom bother, and healthrelated quality of life in patients with overactive bladder taking solifenacin or placebo in the VIBRANT study. Int J Clin Pract. 2011;65(2):211-8.

32. Kubota Y, Kojima Y, Shibata Y, Imura M, Kohri K, Sasaki S. Correlation between improvements in Overactive Bladder Symptom Score and health-related quality of life questionnaires in overactive bladder patients treated with an antimuscarinic drug. Neurourol Urodyn. 2011;30(7):1309-14.
33. Van Kerrebroeck PE, Kelleher CJ, Coyne KS, Kopp Z, Brodsky M, Wang JT. Correlations among improvements in urgency urinary incontinence, health-related quality of life, and perception of bladder-related problems in incontinent subjects with overactive bladder treated with tolterodine or placebo. Health Qual Life Outcomes. 2009;7:13.

34. Brubaker L, Shull B. EGGS for patient-centered outcomes. Int Urogynecol J Pelvic Floor Dysfunct. 2005;16:171-3.

35. Lee KS, Lee YS, Kim JC, Seo JT, Lee JZ, Choo MS. Patient-reported goal achievement after antimuscarinic treatment in patients with overactive bladder symptoms. Int J Clin Pract. 2012;66(7):663-70.

36. Choo MS, Doo CK, Lee KS. Satisfaction with tolterodine: assessing symptom-specific patient-reported goal achievement in the treatment of overactive bladder in female patients (STARGATE study). Int J Clin Pract. 2008;62(2):191-6.

37. Mostafaei H, Janisch F, Mori K, et al. Placebo response in patients with oral therapy for overactive bladder: a systematic review and meta-analysis. Eur Urol Focus. 2021 (Epub ahead of print). 